\title{
Réchauffement climatique et pénurie de médecins-assistants
}

\author{
Jürg Unger-Köppel \\ Dr méd., membre du Comité central de la FMH, responsable du département Médecine et tarifs hospitaliers
}

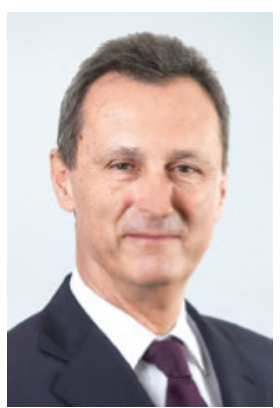

En Argovie, il y a un téléski. A l'époque, les hivers certainement encore très enneigés avaient justifié sa construction. Aujourd'hui, il ne fonctionne presque plus faute de neige, devenue rare dans cette région. D’autres stations de ski, attentives et confrontées à la même évolution, ont commencé à prendre des mesures lorsque cela a été possible et se sont équipées de systèmes d'enneigement artificiel.

Lorsque le téléski argovien a été installé, les médecins qui venaient de réussir l'examen fédéral pouvaient être contents de trouver un poste. A cette époque, les ressources en médecins-assistants étaient aussi abondantes pour les hôpitaux que la neige pour les skieurs. Cela facilitait énormément l'organisation puisque, le plus souvent, si une nouvelle tâche médicale devait être accomplie, elle était simplement attribuée aux médecins-assistants.

\section{L'administration n'est pas un objectif pour les} futurs médecins - ils désirent travailler pour et avec le patient.

La situation sur le marché du travail a ensuite changé; tout d'abord de manière imperceptible pour les médecins. Soudain, les médecins-chefs n'ont plus eu quatre ans d'avance pour engager le meilleur candidat possible. Les médecins-chefs ont progressivement commencé à admettre entre eux qu'ils pouvaient s'estimer heureux d'avoir suffisamment de candidatures pour un poste. Plus tard, on s'est mis à entendre à mots couverts que certains collègues n'arrivaient plus à pourvoir entièrement les postes vacants. En parallèle, l'application de la loi sur le travail a engendré une limitation nette du temps disponible des médecins-assistants. Et avec elle, les ressources en médecins-assistants sont définitivement devenues un facteur limitant de toute l'organisation médicale. Quelles ont été les conséquences d'avoir confié de nombreuses tâches aux médecins-assistants? Plusieurs enquêtes ont montré que le temps accordé au patient est calculé de manière très serrée actuellement. Une majeure partie du temps de travail est consacrée à l'ad- ministration. Mais le travail administratif n'est certainement pas l'objectif de tout nouveau promu, qui vient de passer avec succès le numerus clausus, sinon il devrait être interrogé davantage à ce sujet. Les futurs médecins souhaitent travailler pour et avec les patients. Pour recruter des médecins-assistants, les hôpitaux peuvent aujourd'hui marquer des points s'ils font valoir que leur organisation permet de passer davantage de temps auprès des patients que la concurrence. En concertation avec les équipes médicales, les directions des hôpitaux sont appelées à trouver des voies nouvelles et des solutions pour les questions suivantes: Qu'est-ce qui doit être fait exactement? Quel est le but des tâches administratives? Lesquelles peuvent être totalement abandonnées? Comment retirer aux médecins-assistants (et aux infirmières) les tâches administratives incontournables? Quelles sont les aptitudes requises pour accomplir ces tâches? Quels autres groupes professionnels, dont les compétences clés incluent ces aptitudes, pourraient décharger les médecins? Savoir si une tâche déterminée exige réellement un savoir médical spécifique (ou non) doit être une ligne directrice fondamentale pour répondre à ces questions. Une tâche peut être déléguée à un autre groupe professionnel pour lequel le recrutement est plus simple, si aucune connaissance ni aucun savoirfaire médical ne sont exigés.

L'ASMAC s'est penchée sur ce thème central et a développé différentes idées concrètes pour décharger les médecins-assistants (à la page 1603 du présent nu-

\section{Comment dispenser les médecins-assistants} des tâches administratives? Quels autres groupes professionnels pourraient les en décharger?

méro). Il est recommandé à tous les responsables d'hôpitaux de les examiner. Ces idées de l'ASMAC permettent d'améliorer l'organisation des tâches médicales, à long terme et de manière efficace - et ce tout au long de l'année, à l'inverse des canons à neige dans les stations de ski. 